\title{
A novel wide viewing endoscope for upper gastrointestinal screening: a pilot study
}

Authors

Institution
Naomi Kakushima, Kohei Takizawa, Masaki Tanaka, Noboru Kawata, Sayo Ito, Kenichiro Imai, Kinichi Hotta, Kimihiro Igarashi, Yoshihiro Kishida, Masao Yoshida, Hiroyuki Matsubayashi, Hiroyuki Ono

Division of Endoscopy, Shizuoka Cancer Center, Shizuoka, Japan submitted

4. September 2015

accepted after revision

4. November 2015

\section{Bibliography}

DOI http://dx.doi.org/

$10.1055 / \mathrm{s}-0041-109400$

Published online: 4.2.2016

Endoscopy International Open

2016; 04: E190-E192

(c) Georg Thieme Verlag KG

Stuttgart · New York

E-ISSN 2196-9736

\section{Corresponding author}

\section{Naomi Kakushima, MD PhD}

Division of Endoscopy

Shizuoka Cancer Center

1007 Shimonagakubo

Nagaizumi

Suntougun

Shizuoka 4118777

Japan

Fax: +81-55-9895692

kakushin-tky@umin.ac.jp
The feasibility of full-spectrum endoscopy (FUSE) esophagogastroduodenoscopy (EGD), providing a 245-degree field of view with double imagers on the front and left side of the endoscope was evaluated. Twenty-one healthy individuals (15 male, median age, 35 years) underwent upper gastrointestinal screening using FUSE-EGD. The primary end point was the rate of successful visualization of Vater's papilla. Secondary end points were visualization of the squamo-columnar junction (SCJ) and the anal side of the pyloric ring, and the endoscopists' subjective evaluation of usability based on maneuverability and imaging of FUSE-

\section{Introduction}

$\nabla$

Esophagogastroduodenoscopy (EGD) has become an important examination for screening and diagnosis of diseases of the upper gastrointestinal tract. Improved endoscopic screening was accomplished by the incorporation of a thinner caliber endoscope with the capacity to capture high definition images. However, the ability to visualize Vater's papilla with front viewing endoscopes is less than satisfactory [1]. The duodenum has a narrow lumen; therefore, endoscopes with a side view are preferred for visualization of Vater's papilla, although current side viewing scopes are not suitable for screening in EGD. In addition, switching to a side viewing endoscope may not be a viable option when considering a patient's comfort and the availability of endoscopic equipment.

Full-spectrum endoscopy (FUSE; EndoChoice, Inc., Alpharetta, GA, United States) EGD is a newly developed video-endoscope that provides a 245degree field of view with double imagers on the front and side of the endoscope tip. A wider view for endoscopic examinations, such as ultra-wide viewing scopes, has been reported to enhance diagnostic yield in the field of colonoscopy [2-4]. In the upper gastrointestine, locations with a steep
EGD. The mean procedure time was $6.5 \mathrm{~min}$, with a median of 91 images captured. The rate of successful visualization of Vater's papilla was $90 \%$ (19/21). The whole circumference of the SCJ was observed with two video monitors in all cases. The anterior anal side of the pyloric ring was observed in $29 \%$ (6/21) of cases. However, the general impression of the usability of FUSE-EGD was that it was rather inferior to that of a standard front viewing endoscope. Although the usability requires further modification, FUSE-EGD provided excellent results for imaging Vater's papilla and the SCJ.

curve such as the squamo-columnar junction $(\mathrm{SCJ})$ and the anal side of the pyloric ring may not be well observed by forward viewing endoscopes. Theoretically, the incorporation of double imagers in different directions has a possibility to produce a better view in these locations. However, there are no available data to determine whether a wider endoscopic view is efficient for upper gastrointestinal screening. The aim of this study was to evaluate the feasibility, usability, and safety of FUSE-EGD in human subjects. Feasibility was defined by the completion of taking images according to a standardized protocol within an acceptable time. Usability was defined by technical handling of the endoscope and quality of the images.

\section{Methods and materials \\ $\nabla$}

The study included 21 healthy individuals (15 males and 6 females) with a median age of 35 years (range, 29-55 years) who underwent upper gastrointestinal screening using FUSE-EGD. Each subject received a full explanation with regard to the purpose of this open-label study and gave informed consent for participation in the study. After fasting for more than $12 \mathrm{~h}$, each sub- 

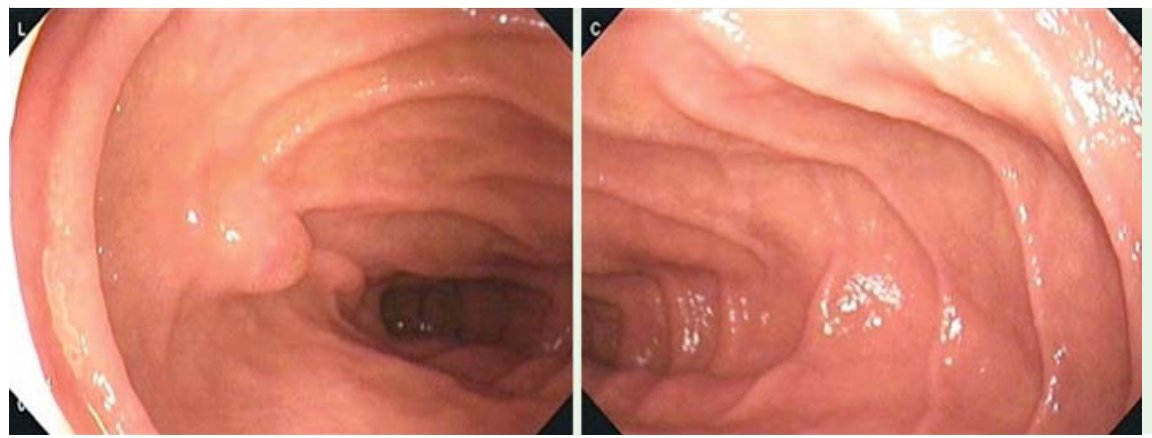

Fig. 1 Visualization of the 2nd portion of the duodenum on two video monitors with a clear view of Vater's papilla.
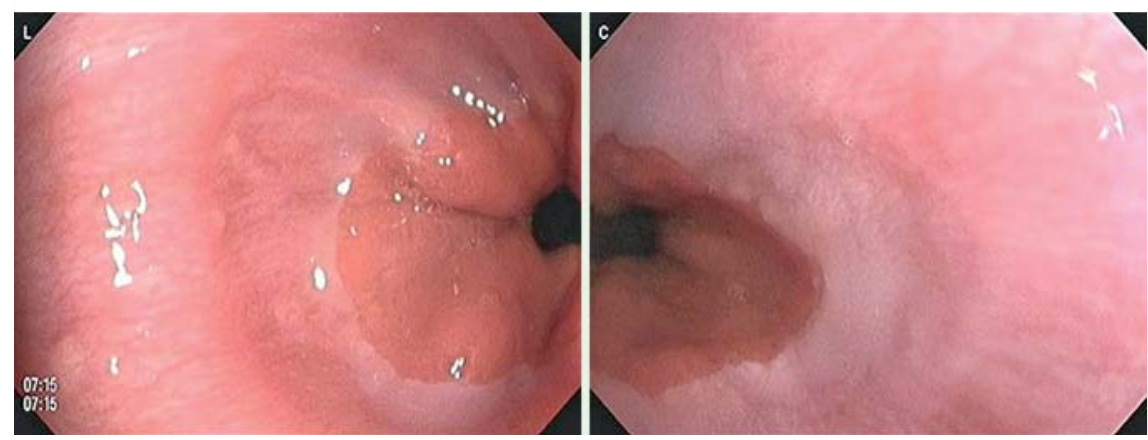

Fig. 2 Visualization of the squamo-columnar junction on the two video monitors of the FUSE-EGD system.

ject drank $100 \mathrm{~mL}$ of water containing pronase, sodium bicarbonate, and simethicone. The examination was performed under oral anesthesia with $1-5$ sprays of $8 \%$ lidocaine, with no sedation. Five endoscopists who were board certified members of the Japanese Gastroenterological Endoscopy Society performed screening of the upper gastrointestine according to a standardized protocol while capturing sequential images from the esophagus to the duodenum. The total duration of the endoscopic procedure was recorded using a stopwatch.

The primary end point of this pilot and feasibility study was the rate of successful visualization of Vater's papilla. Additional end points included the duration of the procedure, successful visualization of the anal side of the pyloric ring and the SCJ, adverse events, and the endoscopists' subjective evaluation of the usability of the FUSE-EGD procedure. The endoscopists' subjective evaluation of the usability, field of view, and maneuverability of FUSE was assessed using a five-point Likert scale with the following answer options: unacceptable, difficult, acceptable, good, and excellent. In addition, the endoscopists' subjective evaluation compared to the standard endoscope used in our hospital $(9.8-\mathrm{mm}$, high definition, front viewing, video endoscope) was also evaluated.

\section{Endoscopy platform}

The FUSE platform consists of a video endoscope and a main control unit. The video images are presented to the endoscopist on two contiguous monitors. The FUSE-EGD (FSG-2500-ST) system is a 9.6- $\mathrm{mm}$, flexible endoscope for repeated clinical use (diagnostic visualization and therapeutic intervention). The endoscope is equipped with a working channel $(2.8 \mathrm{~mm})$, air or $\mathrm{CO}_{2}$ insufflation options, suction, and forward waterjet irrigators. A wide 245-degree field of view is achieved using two lenses and groups of light-emitting diodes positioned on the front and side of the endoscope tip. This retrospective study was approved by the institutional review board of our hospital (No. 27-J69-27-1-3).

\section{Results}

Gastrointestinal screening using the FUSE-EGD system was successfully completed for all 21 subjects with a median procedure time $( \pm \mathrm{SD})$ of $6.5 \pm 1.6 \mathrm{~min}$. No acute or delayed adverse events occurred. A median of $90.6 \pm 24.9$ endoscopic images was acquired. Successful visualization of Vater's papilla was obtained in $90 \%(19 / 21)$ of the subjects ( $\bullet$ Fig. 1 ). The duodenal side of the pyloric ring (anterior wall only) was observed in $29 \%$ (6/21) of the subjects. The whole circumference of the SCJ was observed on the two video monitors in all cases ( $\bullet$ Fig. 2 ). The main finding detected among the subjects was esophageal hernia (100\%, 21/ 21).

Subjective evaluation of the usability of FUSE-EGD was assessed by the five endoscopists, who reported that FUSE-EGD was inferior compared with the standard front viewing endoscope for all participants ( Table 1). However, $80 \%$ of the participants answered that the FUSE-EGD system platform was feasible as a whole.

\section{Discussion}

This is the first study on humans utilizing the newly developed FUSE-EGD for upper gastrointestinal screening. The main end point of this study was to evaluate the feasibility of the new FUSE-EGD system. Successful visualization of Vater's papilla was achieved in $90 \%$ of the study participants. In addition, no adverse events were recorded associated with this system.

The duodenum has a narrow lumen and Vater's papilla is not routinely identified during screening by EGD [1] or by capsule endoscopy [5]. A previous study reported that the detection rate of Vater's papilla in whole or in part was only $57 \%$ using a routine imaging method, but it increased to $89 \%$ using a straightening endoscopic technique [1]. For surveillance of patients with duodenal polyposis, the use of a side viewing endoscope [6] or side and front viewing endoscopes at the same time under general 
Table 1 Qualitative assessment by five endoscopists of the full-spectrum endoscope used for esophagogastroduodenoscopy in 21 subjects.

\begin{tabular}{|ll}
\hline Qualitative aspect & Rated excellent/good/acceptable/difficult/unacceptable, $\%$ \\
\hline General impression & Acceptable: $80 \%$ \\
\hline Ease of scope maneuverability & Good: $20 \%$, acceptable: $80 \%$ \\
\hline Image quality & Acceptable: $20 \%$, difficult: $80 \%$ \\
\hline Field of view & Good: $80 \%$, acceptable: $20 \%$ \\
\hline Brightness & Good: $20 \%$, acceptable: $60 \%$, difficult: $20 \%$ \\
\hline Waterjet irrigation & Good: $20 \%$, acceptable: $60 \%$, difficult: $20 \%$ \\
\hline Usability compared with standard front viewing endoscopy & Inferior: $100 \%$ \\
\hline
\end{tabular}

anesthesia has been reported [7]. However, these complex maneuvers may not be appropriate for upper gastrointestinal screening. The FUSE-EGD system is equipped with a side view imager on a front viewing endoscope that enables easier detection of Vater's papilla with normal manipulation of the endoscope. The FUSE-EGD system allowed easier visualization of Vater's papilla than the conventional endoscope.

The SCJ was visualized on both monitors in all subjects. Although Barrett's esophagus is rare among the Japanese population, a clear view of the entire SCJ is greatly beneficial for the detection of early SCJ and Barrett's adenocarcinoma. Vater's papilla is an excellent marker to assess this system; however, the real clinical benefit could be at the SCJ.

The anal side of the pyloric ring was observed in $29 \%$ of the subjects. We expected that the side lens would visualize the anal side of the pyloric ring. In most cases, the mucosa contacted the side lens, and therefore it was difficult to keep a distance between the mucosa and the lens in the narrow duodenal bulb. The ability to visualize the anal side of the pyloric ring may be beneficial for gastric antral lesions extending to the duodenal bulb; however, these lesions are quite rare.

All of the endoscopists participating in this study reported that the usability of FUSE-EGD was inferior to that of the conventional endoscope. However, all procedures were performed safely and without difficulty with no sedation. Since none of the endoscopists had previous experience using FUSE-EGD, it may just be a matter of getting used to the maneuverability of the scope. The image quality and brightness of the view field are expected to be further improved in the near future.

A limitation to this study was the small sample size and limited number of participating endoscopists. However, the results of this first pilot and feasibility human study of FUSE-EGD are promising.

In conclusion, although its usability requires further modification, FUSE-EGD provided excellent results for imaging Vater's pa- pilla and the SCJ. FUSE-EGD has the potential to improve the efficacy of upper gastrointestinal screening.

\section{Competing interests: None}

\section{Acknowledgments}

We thank our medical engineers, Mr. Shunichi Kondo, Mr. Ryo Shimoda, and Mr. Tatsuya Seo for providing the mechanical support for the equipment. The FUSE system was provided by Adachi, Co., Ltd., Osaka, Japan.

\section{References}

1 Hew WY, Joo KR, Cha JM et al. Feasibility of forward-viewing upper endoscopy for detection of the major duodenal papilla. Dig Dis Sci 2011; 56: 2895-2899

2 Gralnek IM, Carr-Locke DL, Segol 0 et al. Comparison of standard forward-viewing mode versus ultrawide-viewing mode of a novel colonoscopy platform: a prospective, multicenter study in the detection of simulated polyps in an in vitro colon model (with video). Gastrointest Endosc 2013; 77: 472 - 479

3 Gralnek IM, Segol O, Suissa A et al. A prospective cohort study evaluating a novel colonoscopy platform featuring full-spectrum endoscopy. Endoscopy 2013; 45: 697-702

4 Gralnek IM, Siersema PD, Halpern Z et al. Standard forward-viewing colonoscopy versus full-spectrum endoscopy: an international, multicenter, randomized, tandem colonoscopy trial. Lancet Oncol 2014; 15: $353-360$

5 Kong H, Kim YS, Hun JJ et al. Limited ability of capsule endoscopy to detect normally positioned duodenal papilla. Gastrointest Endosc 2006; 64: $538-541$

6 Burke CA, Beck GJ, Church JM et al. The history of untreated duodenal and ampullary adenomas in patients with familial adenomatous polyposis followed in an endoscopic surveillance program. Gastrointest Endosc 1999; 49: 358 - 364

7 Saurin JC, Gutknecht C, Napoleon B et al. Surveillance of duodenal adenomas in familial adenomatous polyposis reveals high cumulative risk of advanced disease. J Clin Oncol 2004; 22: 493-498 